\title{
Global Stability of Large Solutions to the 3D Navier-Stokes Equations
}

\author{
G. Ponce ${ }^{1}$, R. Racke ${ }^{1,2}$, T. C. Sideris ${ }^{1}$, E.S. Titi ${ }^{3,4}$ \\ 1 Department of Mathematics, University of California, Santa Barbara, CA 93106, USA \\ 2 On leave from: Institut für Angewandte Mathematik, Universität Bonn, Wegelerstrasse 10, \\ D-53115 Bonn, Germany \\ 3 Department of Mathematics, University of California, Irvine, CA 92717, USA \\ 4 Center for Applied Mathematics, Cornell University, Ithaca, NY 14853, USA
}

Received: 1 December 1992/in revised form: 7 May 1993

\begin{abstract}
We prove the stability of mildly decaying global strong solutions to the Navier-Stokes equations in three space dimensions. Combined with previous results on the global existence of large solutions with various symmetries, this gives the first global existence theorem for large solutions with approximately symmetric initial data. The stability of unforced $2 \mathrm{D}$ flow under $3 \mathrm{D}$ perturbations is also obtained.
\end{abstract}

\section{Introduction}

It is well known that there are always global weak solutions to the three dimensional Navier-Stokes system

$$
\begin{aligned}
u_{t}-\nu \Delta u+(u \cdot \nabla) u+\nabla p & =f, & & \text { in }[0, \infty) \times \Omega, \\
\nabla \cdot u & =0, & & \text { in }[0, \infty) \times \Omega, \\
u & =0, & & \text { on }[0, \infty) \times \partial \Omega, \\
u(0, \cdot) & =u_{0}, & & \text { in } \Omega .
\end{aligned}
$$

Here, as usual, $u=u\left(t, x_{1}, x_{2}, x_{3}\right)=u(t, x)=\left(u_{1}, u_{2}, u_{3}\right)$ is the velocity vector, $\nu>0$ is the viscosity, $p=p(t, x)$ is the pressure, $f=f(t, x)$ is the external force, and $u_{0}(x)$ is the initial velocity. Equations (1.1)-(1.4) describe the motion of a viscous, incompressible fluid in a domain $\Omega \subset \mathbb{R}^{3}$. Under minimal assumptions on the data $\left(u_{0}, f\right)$, the existence of a weak solution is guaranteed by the results of Leray [9] and Hopf [5]. The uniqueness of $(u, p)$, up to an additive constant for the pressure $p$, remains open in general and is only known for strong solutions which a priori exist locally. The global existence of small strong solutions has been proved, but for large data, strong global solutions are known to exist only under the assumption of certain spatial symmetries. 
Here we shall show that for any global strong solution $(u, p)$ of (1.1)-(1.4) satisfying

$$
\int_{0}^{\infty}\|\nabla u(t)\|^{4} d t<\infty
$$

(where $\|\cdot\|$ denotes the norm in $L^{2}(\Omega)$, the space of square integrable vector fields on $\Omega)$ there is a neighborhood of $\left(u_{0}, f\right)$ such that (1.1)-(1.4) has a global strong solution for any data taken from this neighborhood. The condition (1.5) is a global version of a regularity criterion that goes back to Leray [9]. The idea, roughly speaking, is that if the reference solution decays to zero and another solution is sufficiently close initially, then by local well posedness the perturbed solution should remain close to the reference solution long enough to make small data arguments possible.

From the physical and mathematical point of view, the vorticity

$$
\omega(t, x)=\nabla \times u(t, x),
$$

is an important quantity. The vorticity evolves according to the equation

$$
\omega_{t}+(u \cdot \nabla) \omega-\nu \Delta \omega-(\omega \cdot \nabla) u=\nabla \times f .
$$

The first terms $\omega_{t}+(u \cdot \nabla) \omega$ account for the transport of vorticity along particle trajectories. The effect of the term $\nu \Delta \omega$ is to introduce diffusion, and it is responsible for dissipating the energy and entrophy. However, the term $(\omega \cdot \nabla) u$, the vorticity stretching term, is the one responsible for instabilities and for changing the direction and magnitude of the vorticity. This is the term that one has to control in order to achieve global regularity in the solution.

Indeed, for the 2D Navier-Stokes equations there is no vorticity stretching term, and for this case one has global regularity. (This is still the case for the 2D Euler equation, $\nu=0$.) In particular, one can think of the 2D Navier-Stokes equations as a spatially symmetric solution of the 3D equations. Global strong solutions for the 3D Navier-Stokes equations with large data also exist with axial, rotational, and helical symmetry. Ladyzhenskaya [8] proved the global existence of rotationally symmetric large strong solutions in domains $\Omega$ which are obtained by rotation about the $x_{3}$-axis of a planar domain $D$ lying in the half plane $x_{2}=0, x_{1}>0$ at a positive distance from the $x_{3}$-axis, assuming the angular components of the force $f$ and the initial data $u_{0}$ do not depend on the angle of rotation $\phi$ about the $x_{3}$-axis. Ukhovskii and Iudovich [20] proved the global existence of large strong axially symmetric solutions in the whole space. By axial symmetry is meant that the solution is rotationally symmetric and its component in the $\phi$ direction is zero. Recently, Mahalov, Titi and Leibovich [12] established the existence of unique global strong solutions for large helically symmetric data. There, $\Omega$ is an infinite periodic pipe in the $x_{3}$-direction with Dirichlet boundary conditions on the sides. Helical symmetry means that the solution $u=u\left(r, \phi, x_{3}\right)$ in cylindrical coordinates actually only depends on $r$ and $n \phi+\alpha x_{3}$, where $n \in \mathbb{Z} \backslash\{0\}$ and $\alpha>0$ are given parameters. As in $2 \mathrm{D}$, the axially symmetric flow has no vorticity stretching. Global existence and regularity follow in these cases by estimating directly from the vorticity equation. On the other hand, for the 3D Navier-Stokes with rotational or helical symmetry, the vorticity stretching term is nontrivial. Here, one can take advantage of the symmetry to reduce the spatial dimension of the problem and derive new Sobolev estimates.

One might argue that the symmetry constraint gives the term $(\omega \cdot \nabla) u$ a special form. It is physically significant that one can break the symmetry and still achieve 
global existence. The application of our stability result to the symmetric solutions gives the first existence result for large data which are not symmetric and where the vorticity stretching term can be arbitrarily large. It is worth mentioning that it is an open question whether or not the 3D Euler equation (i.e. $\nu=0$ ) has global regularity in time, even in the presence of symmetry. There are few results in this direction but all of them treat the cases when the vorticity stretching or its equivalent is identically zero. For instance, see [20] which treats axi-symmetric flow. For Euler flow, vortex stretching has been proposed as a mechanism for formation of singularities. For example, in the inviscid case with rotational symmetry, numerical computations [3, 14] indicate the formation of singularities in finite time.

Throughout this paper we will always assume that $\Omega \subset \mathbb{R}^{3}$ is a domain with boundary $\partial \Omega$ uniformly of class $C^{3}$. This means that there exist constants $r, M>0$ such that for any point $x \in \partial \Omega, \partial \Omega \cup B_{r}(x)$ is the graph of a $C^{3}$ function with derivatives up to order 3 bounded by $M$.

Applying the Helmholtz projection $P$,

$$
P: L^{2}(\Omega) \rightarrow H \equiv{\overline{\left\{u \in H_{0}^{1}(\Omega): \nabla \cdot u=0\right\}}}^{L^{2}(\Omega)}
$$

to (1.1)-(1.4) we reformulate the problem as

$$
\begin{aligned}
u_{t}+A u+P((u \cdot \nabla) u) & =P f, \\
u & =P u, \\
\left.u\right|_{\partial \Omega} & =0, \\
u(0, \cdot) & =u_{0},
\end{aligned}
$$

where $A=-\nu P \Delta$ is the Stokes operator with domain

$$
D(A) \equiv H^{2}(\Omega) \cap V, \quad V \equiv H_{0}^{1}(\Omega) \cap H,
$$

where $H^{2}(\Omega)$ and $H_{0}^{1}(\Omega)$ denote the usual Sobolev spaces defined over $\Omega$ (see [1, 19 , or 21$]$.

Our results can be strengthened for domains $\Omega$ which satisfy the Poincaré inequality:

$$
\|g\| \leq C\|\nabla g\|
$$

for all $g \in H_{0}^{1}(\Omega)$. In particular, bounded domains and strips satisfy (1.10).

We can now state our stability result

Theorem 1. Let

$$
v \in L_{\mathrm{loc}}^{\infty}([0, \infty), V) \cap L_{\mathrm{loc}}^{2}([0, \infty), D(A))
$$

be a strong solution of (1.1)-(1.4) with data $v(0, \cdot)=v_{0} \in V, P f_{1}(t, \cdot) \in H$ satisfying

$$
\int_{0}^{\infty}\|\nabla v(t)\|^{4} d t<\infty
$$

(i) Let $\Omega$ be a domain which satisfies (1.10), and let $P f_{1} \in L^{2}([0, \infty), H)$. There is a $\delta>0$ such that if $u_{0} \in V, \operatorname{Pf} \in L^{2}([0, \infty), H)$ with

$$
\left\|\nabla u_{0}-\nabla v_{0}\right\|+\int_{0}^{\infty}\left\|P f(t)-P f_{1}(t)\right\|^{2} d t<\delta,
$$


then there is a unique global strong solution $u$ of (1.6)-(1.9) with data $\left(u_{0}, P f\right)$. Moreover, there is an $M \equiv M(\delta)$ with $M(\delta) \rightarrow 0$ as $\delta \rightarrow 0$ such that

$$
\sup _{t \geq 0}\|\nabla u(t)-\nabla v(t)\| \leq M(\delta),
$$

and, if $\|\nabla v(t)\|$ and $\left\|P f(t)-P f_{1}(t)\right\|$ decay to zero exponentially, then $\|\nabla u(t)\|$ decays to zero exponentially as $t \rightarrow \infty$.

(ii) Let $\Omega \subset \mathbb{R}^{3}$ be a general domain, and let $P f_{1} \in L^{1} \cap L^{2}([0, \infty), H)$. There exists a $\delta>0$ such that if $u_{0} \in V$ and $P f \in L^{1} \cap L^{2}([0, \infty), H)$ with

$$
\left\|u_{0}-v_{0}\right\|_{H^{1}}+\int_{0}^{\infty}\left[\left\|P f(t)-P f_{1}(t)\right\|+\left\|P f(t)-P f_{1}(t)\right\|^{2}\right] d t<\delta,
$$

then there is a unique global strong solution $u$ of (1.6)-(1.9) with data $\left(u_{0}, P f\right)$, and an $M(\delta) \rightarrow 0$ as $\delta \rightarrow 0$ such that

$$
\sup _{t \geq 0}\|u(t)-v(t)\|_{H^{1}} \leq M(\delta) .
$$

A similar abstract stability result in $\mathbb{R}^{n}$ is given in [22], but no attempt is made to identify reference solutions.

We remark that it is possible to allow domains $\Omega$ which rotate at constant speed, since this results in a harmless linear term $\mathbf{b} \times u$ on the right-hand side of Eq. (1.1), for some constant $\mathbf{b} \in \mathbb{R}^{3}$. Theorem 1 carries over to this situation as long as $|\mathbf{b}| / \nu$ is sufficiently small, i.e. slow rotations and/or sufficiently viscous fluids.

The crucial condition (1.11) is satisfied by all known examples of global strong solutions with zero forces. See the examples in Theorem 3 below.

For strong solutions, (1.11) is equivalent to the condition

$$
v \in L^{q}\left([0, \infty), L^{p}(\Omega)\right), \quad \frac{3}{p}+\frac{2}{q}=1, \quad 3<p<\infty,
$$

the local version of which describes a uniqueness and regularity class for weak solutions, $[11,13,16,21,2,17]$. The extremal case, $p=\infty$, can also be included if we replace $L^{\infty}$ by the space of continuous functions vanishing at infinity. We have

Theorem 2. Suppose that $\Omega$ is $\mathbb{R}^{3}$ or a domain for which (1.10) holds, and that the external force is zero. For global strong solutions of (1.1)-(1.4) in the class described in Theorem 1, conditions (1.11) and (1.14) are equivalent.

An application of Theorem 1 to some situations where global solutions are known to exist gives the following:

Theorem 3. (i) Let $\Omega$ be obtained by rotation about the $x_{3}$-axis of a planar domain $D$ lying in the half plane $\left\{x_{2}=0, x_{1}>0\right\}$ at a positive distance from the $x_{3}$-axis. Let $P f_{1}=0$ and suppose that $v_{0} \in D(A)$ is rotationally symmetric, i.e. expressed in cylindrical coordinates $v_{0}$ is independent of the angle of rotation $\phi$ about the $x_{3}$-axis. Then there is a $\delta>0$ such that for any $u_{0} \in V$ and $P f \in L^{2}([0, \infty), H)$ satisfying

$$
\left\|u_{0}-v_{0}\right\|_{H^{1}}+\int_{0}^{\infty}\|P f(t)\|^{2} d t<\delta
$$

there is a unique, global, strong solution $u$ of (1.6)-(1.9). 
(ii) Let $\Omega=\mathbb{R}^{3}$, let $P f_{1}=0$, and suppose that $v_{0} \in D(A) \cap H^{4}\left(\mathbb{R}^{3}\right)$ is axially symmetric, i.e. expressed in cylindrical coordinates $v_{0}$ is independent of the angle of rotation $\phi$ about the $x_{3}$-axis and the component of $v_{0}$ in the $\phi$ direction is zero. Then there is a $\delta>0$ such that for any $u_{0} \in V$ and $P f \in L^{1} \cap L^{2}([0, \infty), H)$ satisfying

$$
\left\|u_{0}-v_{0}\right\|_{H^{1}}+\int_{0}^{\infty}\left[\|P f(t)\|+\|P f(t)\|^{2}\right] d t<\delta,
$$

there is a unique, global, strong solution $u$ of (1.6)-(1.9).

(iii) For $\alpha>0$ and $0<R \leq \infty$, let

$$
\Omega=\left\{\left(x_{1}, x_{2}, x_{3}\right): 0<x_{3}<2 \pi / \alpha, r=\sqrt{x_{1}^{2}+x_{2}^{2}}<R\right\} .
$$

Set (if $R<\infty) \Gamma=\{x \in \Omega: r=R\}$. Let $n \in \mathbb{Z} \backslash\{0\}$ be given and $v_{0}$ be a helical function, i.e. expressed in cylindrical coordinates $v_{0}$ depends only on $r$ and $n \phi+\alpha x_{3}$, with $v_{0} \in H^{1}(\Omega), \nabla \cdot v_{0}=0$, and satisfying the boundary condition

$$
\left.u\right|_{\Gamma}=0, \quad u\left(x_{1}, x_{2}, 0\right)=u\left(x_{1}, x_{2}, 2 \pi / \alpha\right) .
$$

Then there is a $\delta>0$ such that if $P f \in L^{2}([0, \infty), H)$ and $u_{0} \in H^{1}(\Omega)$ with $\nabla \cdot u_{0}=0$, satisfying the boundary conditions (1.15), and

$$
\left\|\nabla u_{0}-\nabla v_{0}\right\|+\int_{0}^{\infty}\|P f(t)\|^{2} d t<\delta,
$$

then there is a unique, global, strong solution $u$ of (1.6), (1.7), (1.9), (1.15).

In the case of two space dimensions, the global existence of strong solutions for arbitrary divergence free initial data was first established by Leray [10]. With a little extra regularity on the inital data, the $2 \mathrm{D}$ solution decays in $L^{\infty}\left(\mathbb{R}^{2}\right)$, see $[6,7,15]$, which permits 3D perturbations of the initial data.

Theorem 4. Let $v_{0}=\left(v_{01}, v_{02}\right) \in L^{1}\left(\mathbb{R}^{2}\right) \cap H^{1}\left(\mathbb{R}^{2}\right)$ with $\nabla \cdot v_{0}=0$. There is a $\delta>0$ such that if $w_{0} \in V$ and $\left\|w_{0}\right\|_{H^{1}\left(\mathbb{R}^{3}\right)}<\delta$, then (1.1)-(1.4) has a unique global strong solution with data $\left(u_{0}, f\right)=\left(\tilde{v}_{0}+w_{0}, 0\right)$, where $\tilde{v}_{0}\left(x_{1}, x_{2}, x_{3}\right)=$ $\left(v_{01}\left(x_{1}, x_{2}\right), v_{02}\left(x_{1}, x_{2}\right), 0\right)$.

Notice that condition (1.11) can not hold for the 2D flow $v(t, \cdot)$ since the $L^{2}$-norm is understood to be in $\mathbb{R}^{3}$. Instead, we measure the decay of the reference solution $v(t, \cdot)$ using $(1.14)$ with $(q, p)=(2, \infty)$. The need for this decay explains the additional requirement that $v_{0} \in L^{1}\left(\mathbb{R}^{2}\right)$. In fact, $v_{0} \in L^{p}$ with $1 \leq p<2$ would suffice.

\section{Proof of the Theorems}

Proof of Theorem 1. Under the assumptions made on $u_{0}$ and $P f$, there exists a local strong solution $u \in L^{\infty}((0, T), V) \cap L^{2}((0, T), D(A))$ of (1.6)-(1.9) for some $T=T\left(\left\|\nabla u_{0}\right\|\right)>0$, (see for example $\left.[1,19]\right)$. This observation shows that in order to extend solutions globally it suffices to control $\|\nabla u(t)\|$ uniformly on the interval of local existence. For this purpose, let $w:=u-v$. Then $w$ satisfies

$$
\left.w_{t}+A w+P[(w \cdot \nabla) w+(w \cdot \nabla) v+(v \cdot \nabla) w)\right]=P f-P f_{1}
$$


with initial value $w(0)=w_{0}=u_{0}-v_{0}$. Observe that $u$ does not appear explicitly in (2.1). Multiplying (2.1) by $A w(t)$ and integrating over $\Omega$, we obtain

$$
\begin{gathered}
\frac{1}{2} \frac{d}{d t}\|\nabla w\|^{2}+\|A w\|^{2}+\underbrace{\int_{\Omega} P((w \cdot \nabla) w) \cdot A w}_{:=I_{1}}+\underbrace{\int_{\Omega} P((w \cdot \nabla) v) \cdot A w}_{:=I_{2}} \\
+\underbrace{\int_{\Omega} P((v \cdot \nabla) w) \cdot A w}_{:=I_{3}}=\underbrace{\int_{\Omega}\left(P f-P f_{1}\right) \cdot A w}_{:=I_{4}}
\end{gathered}
$$

In order to estimate $I_{1}-I_{4}$ we need to collect a few interpolation inequalities for functions $g \in D(A)$. First, under the the assumption that $\partial \Omega$ is uniformly $C^{3}$, it was shown in Lemma 1 of [4] that

$$
\left\|\partial_{i j}^{2} g\right\| \leq C(\|A g\|+\|\nabla g\|)
$$

Since $D(A) \subset P L^{2}(\Omega)$, we may integrate by parts and use Cauchy-Schwarz to get

$$
\begin{aligned}
\|\nabla g\|^{2} & =-\int_{\Omega} \Delta g g=-\int_{\Omega} \Delta g P g \\
& =-\int_{\Omega} P \Delta g P g \leq\|A g\|\|g\| \\
& \leq\|A g\|^{2}+\|g\|^{2} .
\end{aligned}
$$

Therefore, in general, we have the following inequality:

$$
\left\|\partial_{i j}^{2} g\right\| \leq C(\|A g\|+\|g\|) \text {. }
$$

Now in the case where (1.10) holds, it is clear from the argument of (2.3) that

$$
\|g\|+\|\nabla g\| \leq C\|A g\| .
$$

So (2.4) can be improved when $\Omega$ satisfies (1.10) to

$$
\left\|\partial_{i j}^{2} g\right\| \leq C\|A g\| \text {. }
$$

This holds in $\mathbb{R}^{3}$ as well, as can be seen from the Fourier transform.

Next, we have

$$
\|g\|_{L^{6}} \leq C\|\nabla g\|
$$

which holds for any domain $\Omega$. Now by the Gagliardo-Nirenberg inequality in $\mathbb{R}^{3}$, (2.6), and (2.7), we have

$$
\|g\|_{L^{\infty}} \leq C\|g\|_{L^{6}}^{1 / 2}\left\|\partial_{i j}^{2} g\right\|^{1 / 2} \leq C\|g\|_{L^{6}}^{1 / 2}\|A g\|^{1 / 2} \leq C\|\nabla g\|^{1 / 2}\|A g\|^{1 / 2} .
$$

Hence, using the Calderón extension theorem (see for example [18], Chapter 6, Theorem 5) and then (2.4), we obtain for all of the domains under consideration

$$
\begin{aligned}
\|g\|_{L^{\infty}} & \leq C\|g\|_{L^{6}}^{1 / 2}\|g\|_{H^{2}}^{1 / 2} \\
& \leq C\|g\|_{L^{6}}^{1 / 2}\left(\|A g\|^{1 / 2}+\|g\|^{1 / 2}\right) \\
& \leq C\|\nabla g\|^{1 / 2}\left(\|A g\|^{1 / 2}+\|g\|^{1 / 2}\right) .
\end{aligned}
$$


In the case when (1.10), and hence also (2.5), are true, we see that (2.8) holds.

Once again, turning first to the case $\Omega=\mathbb{R}^{3}$, we obtain from Gagliardo-Nirenberg and (2.6)

$$
\|\nabla g\|_{L^{3}} \leq C\|\nabla g\|^{1 / 2}\left\|\partial_{\imath j}^{2} g\right\|^{1 / 2} \leq C\|\nabla g\|^{1 / 2}\|A g\|^{1 / 2}
$$

In the general case, we can apply the Calderón extension theorem and (2.4) to get

$$
\|\nabla g\|_{L^{3}} \leq C\|\nabla g\|^{1 / 2}\left(\|A g\|^{1 / 2}+\|g\|^{1 / 2}\right)
$$

which, exactly as above, improves to (2.10) in the case (1.10) where is available.

Now, we first consider case (i) where (1.10) is valid. Hence, $\Omega$ is such that (2.8) and (2.10) hold. $I_{1}-I_{4}$ are estimated as follows.

$$
\begin{aligned}
\left|I_{1}\right| & \leq C\|w\|_{L^{6}}\|\nabla w\|_{L^{3}}\|A w\| \\
& \leq C\|w\|_{L^{6}}\left(\|\nabla w\|^{1 / 2}\|A w\|^{1 / 2}\right)\|A w\| \\
& \leq C\|\nabla w\|^{3 / 2}\|A w\|^{3 / 2} \\
& \leq C_{\varepsilon}\|\nabla w\|^{6}+\varepsilon\|A w\|^{2}, \quad(\varepsilon>0 \text { arbitrary }) \\
\left|I_{2}\right| & \leq\|w\|_{L^{\infty}}\|\nabla v\|\|A w\| \\
& \leq C\|\nabla v\|\|\nabla w\|^{1 / 2}\|A w\|^{3 / 2} \\
& \leq C_{\varepsilon}\|\nabla v\|^{4}\|\nabla w\|^{2}+\varepsilon\|A w\|^{2} \\
\left|I_{3}\right| & \leq\|v\|_{L^{6}}\left\|\nabla_{w}\right\|_{L^{3}}\|A w\| \\
& \leq C\|\nabla v\|\|\nabla w\|^{1 / 2}\|A w\|^{3 / 2} \\
& \leq C_{\varepsilon}\|\nabla v\|^{4}\|\nabla w\|^{2}+\varepsilon\|A w\|^{2} \\
\left|I_{4}\right| & \leq C_{\varepsilon}\left\|P f-P f_{1}\right\|^{2}+\varepsilon\|A w\|^{2}
\end{aligned}
$$

Choosing $\varepsilon$ sufficiently small, using (2.5), and defining $h(t):=\|\nabla w(t)\|^{2}$, we have proved with (2.2), (2.12)-(2.15) that

$$
h^{\prime}(t)+C_{0} h(t) \leq C\left[h^{3}(t)+\|\nabla v(t)\|^{4} h(t)+\left\|P f(t)-P f_{1}(t)\right\|^{2}\right]
$$

holds with positive constants $C_{0}, C$. Let

$$
\lambda:=C \sup _{s \geq 0} e^{-C_{0} s / 2} \int_{0}^{s} e^{C_{0} \tau / 2}\left\|P f(\tau)-P f_{1}(\tau)\right\|^{2} d \tau .
$$

Observe that $\lambda \leq C \int_{0}^{\infty}\left\|P f(\tau)-P f_{1}(\tau)\right\|^{2} d \tau$ holds. If now

$$
\left\|\nabla w_{0}\right\|^{2}+\lambda \frac{1}{2 \max \left(1, \exp \left\{C \int_{0}^{\infty}\|\nabla v(r)\|^{4} d r\right\}\right)}\left(\frac{C_{0}}{2 C}\right)^{1 / 2}
$$

is true, then we infer that

$$
\|\nabla w(s)\|^{2} \leq\left(\frac{C_{0}}{2 C}\right)^{1 / 2}
$$

holds for $0 \leq s \leq t_{1}$ and some $t_{1}>0$. Thus, (2.16) implies for $s \leq t_{1}$,

$$
h^{\prime}(s)+\frac{C_{0}}{2} h(s) \leq C\left[\|\nabla v(s)\|^{4} h(s)+\left\|P f(s)-P f_{1}(s)\right\|^{2}\right] .
$$


Consequently, by a generalized Gronwall inequality, we obtain

$$
\begin{aligned}
h(s) \leq & e^{-C_{0} s / 2}\left(h(0)+C \int_{0}^{s} e^{C_{0} \tau / 2}\left\|P f(\tau)-P f_{1}(\tau)\right\|^{2} d \tau\right) \\
& \times \exp \left\{C \int_{0}^{s}\|\nabla v(\tau)\|^{4} d \tau\right\},
\end{aligned}
$$

$0 \leq s \leq t_{1}$. Hence

$$
h(s) \leq \frac{1}{2}\left(\frac{C_{0}}{2 C}\right)^{1 / 2}
$$

holds for all $s$ up to $t_{1}$. We conclude that (2.18) holds on the domain of definition of $h$, therefore

$$
\|\nabla w(t)\|^{2} \leq\left(e^{-C_{0} t / 2}\left\|\nabla w_{0}\right\|^{2}+\lambda\right) \exp \left\{C \int_{0}^{\infty}\|\nabla v(\tau)\|^{4} d \tau\right\}
$$

is uniformly bounded by assumption (1.11). $\|\nabla v(t)\|$ is also uniformly bounded as can be seen by multiplying the equation for $v$ by $A v$ and using (2.12), $\int_{0}^{\infty}\left\|P f_{1}(\tau)\right\|^{2} d \tau<$ $\infty$. So we conclude that $\|\nabla u(t)\|$ is uniformly bounded. Thus, $u$ exists globally. The remaining statements in Theorem 1, (i) are now obvious. $\delta$ in (1.12) is chosen according to (2.17).

Now we turn to the general case (ii). Multiplying the differential equation for $w$ by $w(t)$, integrating over $\Omega$ and using

$$
\int_{\Omega} P((w \cdot \nabla) w) \cdot w=\int_{\Omega} P((v \cdot \nabla) w) \cdot w=0,
$$

as well as

$$
\begin{aligned}
\left|\int_{\Omega} P((w \cdot \nabla) v) \cdot w\right| & =\left|\int_{\Omega}(w \cdot \nabla) v \cdot w\right| \\
& \leq C\|w\|_{L^{4}}^{2}\|\nabla v\| \\
& \leq C\|w\|^{1 / 2}\|w\|_{L^{6}}^{3 / 2}\|\nabla v\| \\
& \leq C\|w\|^{1 / 2}\|\nabla w\|^{3 / 2}\|\nabla v\| \\
& \leq C_{\varepsilon}\|\nabla v\|^{4}\|w\|^{2}+\varepsilon\|\nabla w\|^{2},
\end{aligned}
$$

we obtain

$$
\frac{d}{d t}\|w(t)\|^{2}+C_{0}\|\nabla w(t)\|^{2} \leq C\|\nabla v(t)\|^{4}\|w(t)\|^{2}+\left\|P f(t)-P f_{1}(t)\right\|\|w(t)\| .
$$

This implies that

$$
\|w(t)\| \leq\left(\|w(0)\|+\int_{0}^{\infty}\left\|P f(\tau)-P f_{1}(\tau)\right\| d \tau\right) \exp \left\{C \int_{0}^{\infty}\|\nabla v(\tau)\|^{4} d \tau\right\}
$$

for all $t>0$. 
Observing that the estimates for $I_{1}-I_{4}$ in (2.12)-(2.15) literally carry over, except for the addition (in the case when $\Omega \neq \mathbb{R}^{3}$ ), of a term of the form $C\|w\|^{2}$ due to the use of (2.9) and (2.11) instead of (2.8) and (2.10), we obtain

$$
\begin{aligned}
\frac{d}{d t}\|\nabla w(t)\|^{2}+C_{0}\|A w(t)\|^{2} \leq C & {\left[\|\nabla w(t)\|^{6}+\|\nabla v(t)\|^{4}\|\nabla w(t)\|^{2}\right.} \\
& \left.+\|w(t)\|^{2}+\left\|P f(t)-P f_{1}(t)\right\|^{2}\right] .
\end{aligned}
$$

Since

$$
\|\nabla w(t)\|^{2} \leq C\left(\|A w(t)\|^{2}+\|w(t)\|^{2}\right)
$$

this implies

$$
\begin{aligned}
\frac{d}{d t}\|\nabla w(t)\|^{2}+\tilde{C}_{0}\|\nabla w(t)\|^{2} \leq C & {\left[\|\nabla w(t)\|^{6}+\|\nabla v(t)\|^{4}\|\nabla w(t)\|^{2}\right.} \\
& \left.+\|w(t)\|^{2}+\left\|P f(t)-P f_{1}(t)\right\|^{2}\right] .
\end{aligned}
$$

With $h(t):=\|\nabla w(t)\|^{2}$ and $M:=\exp \left\{C \int_{0}^{\infty}\|\nabla v(\tau)\|^{4} d \tau\right\}$ we have using (1.13), (2.20), and (2.21),

$$
h^{\prime}(t)+\tilde{C}_{0} h(t) \leq C\left[h^{3}(t)+\|\nabla v(t)\|^{4} h(t)+\left\|P f(t)-P f_{1}(t)\right\|^{2}+(\delta M)^{2}\right] .
$$

The boundedness of $h(t)$ now follows by Gronwall's inequality, for $\delta$ sufficiently small.

This completes the proof of Theorem 1.

Proof of Theorem 2. Now we turn to the proof of Theorem 2. Assume $v \in$ $L^{q}\left([0, \infty), L^{p}(\Omega)\right)$ for some $p, q$ satisfying $2 / q+3 / p=1, p \in[3, \infty]$.

A standard argument shows that for $\varepsilon>0$,

$$
\frac{1}{2} \frac{d}{d t}\|\nabla v\|^{2}+\|A v\|^{2} \leq C\|(v \cdot \nabla) v\|\|A v\| \leq C_{\varepsilon}\|(v \cdot \nabla) v\|^{2}+\varepsilon\|A v\|^{2} .
$$

Combining the estimates

$$
\begin{aligned}
\|(v \cdot \nabla) v\|^{2} & \leq C\|v\|_{L^{p}}^{2}\|\nabla v\|_{L^{r}}^{2}, & & 1 / p+1 / r=1 / 2, \\
\|\nabla v\|_{L^{r}} & \leq C\|A v\|^{\theta}\|\nabla v\|^{1-\theta}, & & \theta=3 / p=1-2 / q
\end{aligned}
$$

the second of which follows analogously to (2.10) since $2 \leq r \leq 6$, with Young's inequality it follows that

$$
\|(v \cdot \nabla) v\|^{2} \leq C_{\varepsilon}\|v\|_{L^{p}}^{q}\|\nabla v\|^{2}+\varepsilon\|A v\|^{2} .
$$

Now inserting this estimate in the above inequality we infer that

$$
\sup _{t \geq 0}\|\nabla v(t)\|^{2} \leq C\|\nabla v(0)\|^{2} \exp \left(\int_{0}^{\infty}\|v(\tau)\|_{L^{p}}^{q} d \tau\right) .
$$

Hence

$$
\int_{0}^{\infty}\|\nabla v(\tau)\|^{4} d \tau \leq \sup _{t \geq 0}\|\nabla v(t)\|^{2} \int_{0}^{\infty}\|\nabla v(\tau)\|^{2} d \tau<\infty .
$$


Now we assume that

$$
\int_{0}^{\infty}\|\nabla v(t)\|^{4} d t<\infty
$$

The same argument used to estimate $I_{1}$ shows that $\sup _{t \geq 0}\|\nabla v(t)\|^{2}<\infty$ and consequently $\int_{0}^{\infty}\|A v(\tau)\|^{2} d \tau<\infty$. For $p \geq 6$ it follows that

$$
\begin{aligned}
\int_{0}^{\infty}\|v(\tau)\|_{L^{p}}^{q} d \tau & \leq C \int_{0}^{\infty}\|\nabla v(\tau)\|^{q(1 / 2+3 / p)}\|A v(\tau)\|^{q(1 / 2-3 / p)} d \tau \\
& \leq C\left(\int_{0}^{\infty}\|\nabla v(\tau)\|^{\alpha q(1 / 2+3 / p)} d \tau\right)^{1 / \alpha}\left(\int_{0}^{\infty}\|A v(\tau)\|^{2} d \tau\right)^{1 / \beta}
\end{aligned}
$$

with $a=4 / q$, therefore $\alpha q(1 / 2+3 / p) \geq 2$ which completes the proof for $p \geq 6$.

The proof of the case $p<6$ combines the previous argument with the inequality

$$
\|v\|_{L^{p}} \leq C\|v\|^{\theta}\|v\|_{L^{6}}^{1-\theta}
$$

where $\theta=3 / p-1 / 2$.

Proof of Theorem 3. Theorem 3 follows immediately from Theorem 1. Part (i) depends on Theorem 6 of [8], p. 162, which guarantees the global existence of rotationally symmetric strong solutions $v$ with $\nabla v \in L^{2} \cap L^{\infty}\left([0, \infty), L^{2}(\Omega)\right)$ which implies (1.11).

Part (ii) depends on the global existence result Theorem 3.1 for axially symmetric solutions in [20]. Notice that $v_{0} \in H^{4}\left(\mathbb{R}^{3}\right)$ is sufficient to satisfy the hypotheses of [20]. Equation (1.11) is verified by showing again that $\nabla v \in L^{\infty}\left([0, \infty), L^{2}(\Omega)\right)$. This follows by an energy estimate for the scalar vorticity equation (1.6) in [20], using the bounds (1.5) and (1.12) of [20] as well as an interpolation inequality for cylindrically symmetric functions.

Part (iii) follows analogously from Theorem 1 (i) by using the global existence results of [12] (for zero forces and no rotation $b=0$ ) observing that Theorem 1 carries over to the boundary conditions (1.15). For the case where the domain $\Omega$ is a periodic strip, i.e. $\Omega=\left\{\left(x_{1}, x_{2}, x_{3}\right): 0 \leq x_{3} \leq 2 \pi / \alpha\right\}$, with periodic boundary conditions, we mention that the ideas of [12] can be also applied to construct a global solution in the appropriate class to which part (ii) of Theorem 1 applies.

Proof of Theorem 4. Let $v=\left(v_{1}, v_{2}\right)$ be a strong solution of the 2D Navier-Stokes equation with data $v_{0}=\left(v_{01}, v_{02}\right) \in V\left(\mathbb{R}^{2}\right) \cap L^{1}\left(\mathbb{R}^{2}\right)$. Thus, we have

$$
v \in L^{\infty}([0, \infty), V) \cap L_{\mathrm{loc}}^{2}([0, \infty), D(A)) .
$$

By the smoothing properties of the Navier-Stokes equation, we know that

$$
\Delta v(t) \in L^{2}\left(\mathbb{R}^{2}\right) \text { for all } t>0,
$$

and from the vorticity equation in two dimensions we also know that

$$
\int_{0}^{\infty}\|\Delta v(\tau)\|^{2} d \tau<\infty
$$


Moreover, using this estimate and the decay of solutions $[15,7,6]$,

$$
\left\|\partial_{x}^{j} v(t)\right\|_{L^{p}\left(\mathbb{R}^{2}\right)}<C t^{-d}
$$

where $d=\min \left\{1^{-}, 1 / p^{\prime}-1 / p+j / 2\right\}, p \in[2, \infty], \frac{1}{p}+\frac{1}{p^{\prime}}=1$, and $j=0,1$, it follows that

$$
\int_{0}^{\infty}\|\nabla v(\tau)\|_{L^{4}\left(\mathbb{R}^{2}\right)} d \tau<\infty
$$

and

$$
\int_{0}^{\infty}\|v(\tau)\|_{L^{\infty}\left(\mathbb{R}^{2}\right)} d \tau<\infty .
$$

We are going to construct a global solution of the form $u=\tilde{v}+w$, where $\tilde{v}=\left(v_{1}, v_{2}, 0\right)$ and $w$ is small in $V\left(\mathbb{R}^{3}\right)$. The equation for $w$ is again given by

$$
w_{t}+A w+P[(w \cdot \nabla) w+(w \cdot \nabla) \tilde{v}+(\tilde{v} \cdot \nabla) w]=0 .
$$

We claim that for $w(t) \in V\left(\mathbb{R}^{3}\right)$ and $\tilde{v}(t)$ as above, the terms $\tilde{v} \cdot \nabla w$ and $w \cdot \nabla \tilde{v}$ lie in $L^{2}\left(\mathbb{R}^{3}\right)$ for positive times, and hence, it makes sense to apply the three-dimensional Helmoltz projection to them. An application of Hölder's inequality is enough to bound the first term:

$$
\|\tilde{v} \cdot \nabla w\| \leq\|v\|_{L^{\infty}\left(\mathbb{R}^{2}\right)}\|\nabla w\| .
$$

The second term is estimated by considering the variables $x_{1}, x_{2}$ separately from $x_{3}$ :

$$
\left\|w \cdot \nabla \tilde{v}\left(\cdot, x_{3}\right)\right\|_{L^{2}\left(\mathbb{R}^{2}\right)}^{2} \leq\left\|w\left(\cdot, x_{3}\right)\right\|_{L^{4}\left(\mathbb{R}^{2}\right)}^{2}\|\nabla v\|_{L^{4}\left(\mathbb{R}^{2}\right)} .
$$

Using the Gagliardo-Nirenberg inequality in two dimensions we have that

$$
\left\|w\left(\cdot, x_{3}\right)\right\|_{L^{4}\left(\mathbb{R}^{2}\right)}^{2} \leq\left\|\nabla_{x_{1}, x_{2}} w\left(\cdot, x_{3}\right)\right\| w\left(\cdot, x_{3}\right) \|_{L^{2}\left(\mathbb{R}^{2}\right)},
$$

so if we integrate with respect to $x_{3}$ and use Cauchy-Schwarz, we obtain

$$
\|w \cdot \nabla \tilde{v}\| \leq\|\nabla w\|^{1 / 2}\|w\|^{1 / 2}\|\nabla v\|_{L^{4}\left(\mathbb{R}^{2}\right)} .
$$

With this observation, the equation for $w$ can be solved locally in $V\left(\mathbb{R}^{3}\right)$ in a straightforward manner employing, for example, its equivalent formulation as an integral equation and standard estimates for the linear heat equation. Thus, it is enough to get an a priori bound for $\|w(t)\|_{H^{1}}$. This will be done in a fashion similar to the proof of Theorem 1 part (ii).

Multiplying (2.25) by $w$ and using (2.19) again leads to

$$
\frac{1}{2} \frac{d}{d t}\|w\|^{2}+\nu\|\nabla w\|^{2}+\int_{\mathbb{R}^{3}}(w \cdot \nabla) \tilde{v} \cdot w=0 .
$$

After an integration by parts and an application of Young's inequality, this last integral is bounded by $C\|v\|_{L^{\infty}}^{2}\|w\|^{2}+\varepsilon\|\nabla w\|^{2}$. We obtain the differential inequality

$$
\frac{d}{d t}\|w\|^{2}+C_{0}\|\nabla w\|^{2} \leq C\|v\|_{L^{\infty}}^{2}\|w\|^{2}
$$


which immediately gives the bound

$$
\|w(t)\|^{2} \leq\|w(0)\|^{2} \exp \left\{C \int_{0}^{t}\|v(\tau)\|_{L^{\infty}}^{2} d \tau\right\} \leq C\|w(0)\|^{2} .
$$

On the other hand, multiplication by $A w(t)$ yields

$$
\frac{1}{2} \frac{d}{d t}\|\nabla w\|^{2}+\|A w\|^{2} \leq\left|I_{1}\right|+\left|I_{2}\right|+\left|I_{3}\right|
$$

as in (2.2). Now

$$
\left|I_{1}\right| \leq C\|\nabla w\|^{6}+\varepsilon\|A w\|^{2}
$$

just as in (2.12). By (2.27), we get

$$
\begin{aligned}
\left|I_{2}\right| & \leq\|(w \cdot \nabla) v\|\|A w\| \\
& \leq\|\nabla w\|^{1 / 2}\|w\|^{1 / 2}\|\nabla v\|_{L^{4}\left(\mathbb{R}^{2}\right)} \mid\|A w\| \\
& \leq C\|w\|^{2}+C\|\nabla v\|_{L^{4}\left(\mathbb{R}^{2}\right)}^{4}\|\nabla w\|^{2}+\varepsilon\|A w\|^{2}
\end{aligned}
$$

and by (2.26),

$$
\left|I_{3}\right| \leq C\|v\|_{L^{\infty}}^{2}\|\nabla w\|^{2}+\varepsilon\|A w\|^{2} .
$$

Therefore, we have deduced the inequality

$$
\begin{aligned}
& \frac{d}{d t}\|\nabla w\|^{2}+C_{0}\|A w\|^{2} \\
& \quad \leq C\left[\|\nabla w\|^{6}+\left(\|v\|_{L^{\infty}}^{2}+\|\nabla v\|_{L^{4}\left(\mathbb{R}^{2}\right)}^{4}\right)\|\nabla w\|^{2}+\|w\|^{2}\right] .
\end{aligned}
$$

It follows from (2.23), (2.24), (2.28), and (2.29) that $\|\nabla w(t)\|^{2}$ remains bounded if $\left\|w_{0}\right\|_{H^{1}}$ is sufficiently small as in the proof of Theorem 1 (ii).

Acknowledgements. We would like to thank T. Miyakawa for helpful discussions concerning inequalities (2.4) and (2.6). G. Ponce and T.C. Sideris are partially supported by the National Science Foundation. R. Racke was partially supported by the Sonderforschungsbereich 256 of the Deutsche Forschungsgemeinschaft. E.S. Titi is supported in part by a grant from the Air Force Office of Scientific Research, \#F49620-92-J-0287 and by the National Science Foundation. Part of this work was done while E.S. Titi was visiting the CNLS and T-Division of the Los Alamos National Lab.

\section{References}

1. Constantin, P., Foias, C.: Navier-Stokes equations. Lectures in Mathematics. Chicago: The University of Chicago Press, 1988

2. Giga, Y.: Regularity criteria for weak solutions of the Navier-Stokes system. Proc. Symposia in Pure Math. 45, Providence, RI: AMS 1986, pp. 449-453

3. Grauer, R., Sideris, T. C.: Numerical computation of 3D incompressible ideal fluids with swirl. Phys. Rev. Lett. 67, 3511-3514 (1991)

4. Heywood, J.G.: The Navier-Stokes equations: On the existence, regularity and decay of solutions. Indiana Univ. Math. J. 29, 639-681 (1980)

5. Hopf, E.: Über die Anfangswertaufgabe für die hydrodynamischen Grundgleichungen. Math. Nachr. 4, 213-231 (1951)

6. Kajikiya, R., Miyakawa, T.: On $L^{2}$ decay of weak solutions of the Navier-Stokes equations in $\mathbb{R}^{n}$. Math. Z. 192, 135-148 (1986) 
7. Kato, T.: Strong $L^{p}$-solutions of the Navier-Stokes equation in $\mathbb{R}^{m}$, with applications to weak solutions. Math. Z. 187, 471-480 (1984)

8. Ladyzhenskaya, O. A.: The mathematical theory of viscous incompressible flow. New York: Gordon and Breach Science Publishers, 1969

9. Leray, J.: Sur le mouvement d'un liquide visqueux emplissant l'espace. Acta Math. 63, 193-248 (1934)

10. Leray, J.; Étude de diverses équations intégrales non linéares et de quelques problèmes que pose l'Hydrodynamique. J. Math. Pure Appl. 12, 1-82 (1933)

11. Lions, J. L.: Sur la regularité et l'unicité des solutions turbulentes des équations de Navier Stokes. Rend. Sem. Mat. Padova, 30, 16-23 (1960)

12. Mahalov, A., Titi, E. S., Leibovich, S.: Invariant helical subspaces for the Navier-Stokes equations. Arch. Rat. Mech. Anal. 112, 193-222 (1990)

13. Prodi, G.: Un teorema di unicita per le equazioni di Navier-Stokes. Annali di Mat. 48, 173-182 (1959)

14. Pumir, A., Siggia, E.: Development of singular solutions to the axisymmetric Euler equations. Phys. Fluids A 4, 1472-1491 (1992)

15. Schonbek, M. E.: Large time behaviour of solutions to the Navier-Stokes equations. Comm. P. D. E. 11, 733-763 (1986)

16. Serrin, J.: The initial value problem for the Navier-Stokes equations. Nonlinear Problems, R. E. Langer, (ed.), Madison, Wis.: University of Wisconsin Press, 1963, pp. 69-98

17. Sohr, H.: Zur Regularitätstheorie der instationären Gleichungen von Navier-Stokes. Math. Z. 184, 359-375 (1983)

18. Stein, E. M.: Singular integrals and differentiability properties of functions. Princeton NJ: Princeton University Press 1970

19. Temam, R.: The Navier-Stokes equations. Theory and numerical analysis. Amsterdam: NorthHolland 1979

20. Ukhovskii, M.R, Iudovich, V.I.: Axially symmetric flows of ideal and viscous fluids filling the whole space. J. Appl. Math. Mech. 32, 52-62 (1968)

21. von Wahl, W.: The equations of Navier-Stokes and abstract parabolic equations. Aspects of Mathematics, E8. Braunschweig/Wiesbaden: Friedr. Vieweg \& Sohn 1985

22. Wiegner, M.: Decay and stability in $L_{p}$ for strong solutions of Cauchy problem for the NavierStokes equations. The Navier-Stokes equations. Theory and numerical methods. Proceedings Oberwolfach (1988), eds. J.G. Heywood, et. al, Lect. Notes Math. 1431, Berlin, Heidelberg, New York: Springer 1990, pp. 95-99

Communicated by A. Jaffe 
